\title{
EP-211
}

\section{Vascular involvement assessment in patients with hilar cholangiocarcinoma by 128-row multidetector computed tomography}

\author{
Julia KULEZNEWA, Boris NIKITIN, Natalia ORLOVA, Konstantin LESKO*
}

Radiology Department, The Loginov Moscow Clinical Scientific Center, Moscow, Russia

Introduction: The purpose was to evaluate the diagnostic accuracy of multidetector 128-row computed tomography (MDCT) in tumor-vessel contact assessment for predicting vascular invasion in patients with hilar cholangiocarcinoma (HCCA).

Methods: We analyzed results of MDCT with intravenous contrast enhancement in 29 patients with MDCT-pattern of HCCA who underwent surgical treatment and verified HCCA from January 2015 to July 2019. We assessed tumor-vessel contact in degrees of the circumference and length of contact in combination and separately. Hepatic arteries (HA) and portal vein (PV) involvement assessed separately. There were 49 cases of potential HA involvement and 48 cases of potential PV involvement.

Results: We expected vascular involvement, when at least one sign was positive: tumor-vessel contact $\geq 180^{\circ}$ and/or length of tumor-vessel contact $\geq 11 \mathrm{~mm}$. Sensitivity (Sn), specificity (Sp), accuracy (Ac), positive (PPV) and negative predictive value (NPV) of combined approach were $90.3 \%, 66.7 \%, 81.6 \%, 82.4 \%$ and $80 \%$ for tumor-HA contact and 96.8\%, 70.6\%, 87.7\%, 85.7\%, 92.3\% for tumor-PV contact, respectively. Tumor-vessel contact circumference assessment approach had Sn 83.9\%, Sp 88.8\%, Ac 85.7\%, PPV 96.9\%, NPV 76.2\% for tumor-HA contact and Sn 90.3\%, Sp 88.2\%, Ac 89.6\%, PPV 93.3\%, NPV 83.3\% for tumor-PV contact; tumor-vessel contact length assessment had Sn 82.1\%, Sp 66.7\%, Ac 76.1\%, PPV 79.3\%, NPV 70.6\% for tumor-HA contact and Sn 78.1\%, Sp 81.2\%, Ac 79.2\%, PPV 89.3\%, NPV 65\% for tumor-PV contact.

Conclusions: Combined approach was more sensitive, but less specific and accurate than tumor-vessel contact circumference assessment. Thus, tumor-vessel contact circumference assessment is more suitable than combined approach. 